\title{
Inteligencia emocional con dispositivos móviles: un análisis de apps para niños en edad infantil
}

\author{
Emotional intelligence with mobile devices: An analysis of apps for \\ children in early childhood
}

\author{
Susana Franco Hernández \\ Universidad de Murcia \\ susana.franco1@um.es \\ Isabel Mํㅗ Solano Fernández \\ Universidad de Murcia \\ imsolano@um.es
}

Recibido: 31/10/2017

Aceptado: $28 / 12 / 2017$

Publicado: 31/12/2017

\section{RESUMEN}

La gran difusión de los dispositivos móviles en los hogares está propiciando la aparición de nuevos recursos que pueden suponer una oportunidad de aprendizaje, ya no sólo enfocados al desarrollo de las capacidades propias del aprendizaje cognitivo sino también al desarrollo emocional de los niños y a la consolidación de sus valores, contribuyendo así a su desarrollo integral.

En esta investigación de tipo evaluativo se realiza un análisis de algunas aplicaciones educativas para dispositivos móviles que permiten trabajar las emociones en niños en edad infantil, de 2 a 8 años. La recogida de información y el análisis de dichas aplicaciones se llevan a cabo por medio de una ficha de registro de información elaborada y validada para tal fin, que ha sido publicada con licencia creative commons para favorecer su reutilización. Los resultados presentan la información obtenida para cada aplicación en relación con las dimensiones de la inteligencia emocional (intrapersonales; interpersonales; manejo del estrés y capacidad de adaptación) y las características técnicas y de diseño (composición general del contenido, con concreción en las instrucciones y adaptación al usuario).

\section{PALABRAS CLAVE}

Inteligencia emocional; Infancia; Tecnología; dispositivos móviles; apps.

\begin{abstract}
The great diffusion of mobile devices in households is encouraging the emergence of new resources that can be a learning opportunity, already not only focused on the development of cognitive learning capabilities but also to the emotional development of children and to the consolidation of its values, thus contributing to the integral development.

In this type of evaluative research is an analysis of some educational applications for mobile devices that allow you to work the emotions in children age 2 to 8 years. The collection of information and analysis of these applications are conducted by means of a registration form of information elaborated and validated for this purpose, which has been published by creative commons license to encourage its reuse. The results present information obtained for each application in relation to the dimensions of emotional intelligence (intrapersonal; interpersonal; stress management and adaptability) and features technical and design) general composition of the content, with concretion in the instructions and adaptation to the user).
\end{abstract}

\section{KEYWORDS}

Emotional Intelligence; early childhood; Technology; mobile devices; apps.

\section{CITA RECOMENDADA}

Franco, S. y Solano, I. (2017). Título del artículo Inteligencia emocional con dispositivos móviles: un análisis de apps para niños en edad infantil. RIITE. Revista Interuniversitaria de Investigación en Tecnología Educativa, 3, 51-63. Doi: http://dx.doi.org/10.6018/riite/2017/308641 


\section{Principales aportaciones del artículo y futuras líneas de investigación:}

- El artículo presenta un tema actual de carácter interdisciplinar, de los ámbitos de la Psicología de la Educación y la Tecnología Educativa.

- Por la versatilidad en la que se han presentado los resultados, así como el instrumento usado para el análisis de las app, puede servir tanto para el contexto formal (maestros de infantil y primaria), como los contextos no formales e informales, principalmente padres, entidades y empresas destinadas al cuidado y entretenimiento infantil.

- Una de las principales aportaciones del artículo reside en la construcción de un instrumento, validado mediante juicio de expertos, y testado mediante el análisis de las aplicaciones que se presentan en el artículo.

- El instrumento ha sido publicado con licencia Creative Commons de reconocimiento y compartir de la misma forma. Ello permite que pueda ser usado y modificado por otros, de forma que las modificaciones y resultados obtenidos puedan ser puesto al servicio de la comunidad científica que lo considere de utilidad. De esta forma enriqueceremos la visión con la que fue construido y validado el instrumento.

- Se podría realizar un análisis más completo de apps de inteligencia emocional por parte de diferentes agentes educativos: expertos en contenidos, expertos del ámbito de la pedagogía, expertos de la psicología, expertos de la tecnología, padres, profesionales en ejercicio, etc.

- Se podría calificar el potencial educativo de las app analizadas a través de una evaluación por juicio de expertos usando el método Delphi.

\section{INTRODUCCIÓN}

Según los datos del último informe Spain Digital Future in Focus de ComScore (2015) España lidera el puesto europeo en cuanto a mayor penetración de smartphones, con aproximadamente 7 millones de unidades vendidas. En el informe se constata además que, incluso los niños de 2 y 3 años ya acceden habitualmente a los smartphones y tablets de sus padres, utilizando aplicaciones para jugar, dibujar, colorear o ver series infantiles de televisión. Acciones todas ellas que pueden entenderse como una oportunidad para su educación (Canovas, 2014). En 2016, se realizó un estudio europeo acerca del uso de la tecnología y los procesos cognitivos que éste desencadena a edades tempranas. Este proyecto presenta un estudio comparativo a nivel europeo que analiza cómo los menores utilizan Internet y los nuevos dispositivos digitales en el ámbito familiar. Una de las conclusiones del informe constata que los padres entienden el acceso temprano a la tecnología como un proceso de familiarización y de preparación para el futuro de sus hijos, en los ámbitos sociales, escolares y laborales (Matsumoto et al, 2016).

La información recogida por el Instituto Nacional de Estadística en el año 2016, en su Encuesta sobre Equipamiento y Uso de Tecnologías de Información y Comunicación en los Hogares, muestra la tendencia al alza de conexión a internet de los hogares españoles, que podría vincularse al uso progresivo de dispositivos móviles y al aumento de la utilización de las tecnologías de la información por la población infantil.

El conocido estudio americano Nielsen State of the Media: The Social Media Report 2012, realizado con una muestra de 1998 usuarios de redes sociales, concluyó que el tiempo dedicado al uso del PC aumentó un 4\% y, en el caso de los Smartphone, un $22 \%$. Además, se duplicó el tiempo de uso de aplicaciones móviles (que subió un 120\%). El mercado responde a esta tendencia multiplicando también el número de aplicaciones disponibles (Crescenzi, 2014).

Según León (2012), las tablets están pasando a ocupar un lugar privilegiado como herramienta de aprendizaje y ocio para más de una generación. Contemplamos el impacto en el desarrollo cognitivo con variables como la hiperconectividad conceptual, el aumento de la 
información visual y su influencia sobre el razonamiento abstracto, los potenciales cambios sobre el desarrollo visoespacial, los paradigmas del aprendizaje humano, etc. Algunas investigaciones describen el aprendizaje mediado por las tecnologías como un proceso beneficioso en ya que propicia el autoconcepto y las actitudes beneficiosas para el aprendizaje (Van, Ellis y Railsback, 2001) llegando a constatar mediante diferentes estudios (Segers, Takke, y Verhoeven, 2004) cómo a través de recursos tecnológicos se puede propiciar en los niños la adquisición del lenguaje, de vocabulario y la comprensión lectora entre otras competencias. Igualando, al menos, los efectos de éstos, a la interacción en un proceso de aprendizaje, de un adulto con un niño (Silverman y Hines, 2009)

Prensky (2006) hace alusión al planteamiento del doctor Bruce D. Berry, de la Universidad de Medicina de Baylor, quien afirma que los avances tecnológicos en los que se han visto inmersas las generaciones nacidas en las últimas décadas pueden llegar a cambiar las estructuras cerebrales y por tanto los modos de pensar y procesar la información. Estos niños han nacido y crecido rodeados de ordenadores, vídeos y videojuegos, música digital, telefonía móvil y otros entretenimientos y herramientas digitales.

Este panorama de uso temprano de la tecnología por parte de la población infantil se puede ver enriquecido con el tratamiento de las emociones por medio de estas tecnologías. Según Kelly (2012), la mayoría de las investigaciones sobre aprendizaje demuestran que las personas aprenden mejor en presencia de algún tipo de conexión emocional con los contenidos que se están aprendiendo. En la entrevista llevada a cabo por Rob Kelly a Rick Van Sant (2009), en base a sus investigaciones más recientes, afirma: "Una de las cosas que sabemos sobre el aprendizaje es que el aprendizaje de la emoción es una experiencia mucho más profunda que el aprendizaje sin emoción" (p.1).

Mediante el uso de la tecnología cualquier material educativo puede adquirir un mayor significado emocional, sobre todo tratándose de temas abstractos, como apunta Negroponte (1995). Este autor asegura que a día de hoy, por medio de un ordenador, es posible la simulación de cualquier cosa, "no hace falta disecar a una rana para estudiarla" (p.120). Es posible jugar con la información, se pueden diseñar ranas, construir un animal con un comportamiento como el de una rana, modificar su comportamiento, simular sus músculos o jugar con ella. Así puede conectarse con el sentimiento de las personas para mejorar su aprendizaje emocional (Chia-Jung Lee, 2011).

Aunque las herramientas digitales para apoyar el aprendizaje social y emocional siguen siendo escasas, en este momento existen instituciones que las están desarrollando para el tratamiento de las emociones. Como apunta Furger (2011), éste es el caso del Teachers College de la Universidad de Columbia, donde la profesora Stern está trabajando con sus alumnos en desarrollar esta clase de herramientas recopilándolas en un sitio web, donde se incluyen juegos, actividades y apps diseñadas para alentar a niños y jóvenes a explorar sus sentimientos.

Las tablet son dispositivos que transciende a lo lúdico, a aquello que incide en nuestras emociones que, al igual que los sentimientos, puede fomentar el aprendizaje (De La Barrera y Donolo, 2009) porque potencian la actividad de las redes neurales y las conexiones sinápticas. Goleman (1996) manifiesta que se puede construir conocimiento, tanto desde la mente racional como mediante la emocional. Ambas están absolutamente interrelacionadas y tienen un rol vital en el desarrollo del aprendizaje.

A día de hoy están aumentando cada vez más los portales o catálogos en Internet que seleccionan y efectúan un análisis de aplicaciones educativas en función de la oferta de los buscadores de App Store ${ }^{1}$ y Google Play ${ }^{2}$. En la tabla siguiente se presentan algunos de los catálogos más relevantes y su criterio de clasificación de apps.

\footnotetext{
1 Portal de App Store: https://itunes.apple.com/

2 Portal de Google Play: https://play.google.com/
} 
Tabla 1. Portales y catálogos de búsqueda de apps para niños. Elaboración propia.

\begin{tabular}{|c|c|c|c|c|}
\hline Sitio web & $\begin{array}{l}\text { № de } \\
\text { apps }\end{array}$ & $\begin{array}{l}\text { Soporte del } \\
\text { software }\end{array}$ & $\begin{array}{l}\text { Criterios de } \\
\text { clasificación }\end{array}$ & URL \\
\hline Eduapps & Alto & iOs & $\begin{array}{l}\text { Etapa educativa, materia, } \\
\text { bloque y currículo }\end{array}$ & http://www.eduapps.es/ \\
\hline Frikids & Alto & Android/iOS/Ebooks & Intereses, edad e idioma & http://www.frikids.com/ \\
\hline $\begin{array}{l}\text { Top best } \\
\text { apps for kids }\end{array}$ & Bajo & Android/iOS/Kindle & Edad & http://topbestappsforkids.com/ \\
\hline Pequetablet & Medio & Android/iOS & $\begin{array}{l}\text { Etapa educativa y } \\
\text { temática }\end{array}$ & http://pequetablet.com/ \\
\hline Edshelf & Alto & Android/iOS/Web & $\begin{array}{l}\text { Precio, edad, materia y } \\
\text { temática }\end{array}$ & https://edshelf.com/ \\
\hline $\begin{array}{l}\text { Proyecto } \\
\text { Guappis }\end{array}$ & Medio & Android/iOS & $\begin{array}{l}\text { Temática, etapa } \\
\text { educativa y capacidades }\end{array}$ & $\begin{array}{l}\text { http://proyectoguappis.blogspo } \\
\text { t.com.es/ }\end{array}$ \\
\hline $\begin{array}{l}\text { Educational } \\
\text { App Store }\end{array}$ & Medio & $\begin{array}{l}\text { Android/los } \\
\text { Windows Phone }\end{array}$ & Precio, temática y edad & $\begin{array}{l}\text { http://www.educationalappstor } \\
\text { e.com }\end{array}$ \\
\hline Appcrawlr & Alto & $\begin{array}{l}\text { Android/iOS/ } \\
\text { Windows Phone }\end{array}$ & $\begin{array}{l}\text { Precio, temática y } \\
\text { categoría. }\end{array}$ & http://appcrawlr.com/ \\
\hline
\end{tabular}

Estos sitios webs se centran solamente en el desarrollo cognitivo del niño, obviando la importancia del desarrollo emocional. Siendo este complemento indispensable del desarrollo cognitivo y, constituyendo ambos, cognitivo y emocional, elementos principales para el proceso de desarrollo integral de la personalidad (Bisquerra, 2000).

Educar emocionalmente puede ayudar a las personas a desarrollar y disfrutar de una situación más favorable en todas las esferas de la vida (Goleman, 1996). Algunos estudios están demostrando cómo el estar en posesión de un alto grado de inteligencia emocional es, al menos, tan importante como tener un $\mathrm{Cl}$ elevado (Extremera y Fernández-Berrocal, 2003). Existe una verdadera relación entre el aprendizaje emocional y las funciones educativas de los medios, funciones tales como la Formativa Global que defiende Zabalza (1987), la cual está relacionada con los valores educativos que el medio transmite, aquellos efectos, instructivos o no, del uso del propio medio. Hoy día se idenfica al aula escolar como el medio de aprendizaje por excelencia. Sin embargo, según Roig (2011) no se deben menospreciar aquellos recursos que se utilicen fuera de ésta porque provengan de ámbitos no formales o hayan sido conformados para el ocio. En la mayoría de los casos estos recursos poseen un gran poder de captación y a partir de ellos se puede enseñar a construir, crear, comprender, interactuar y aprender.

Para demostrar lo inherente de la relación entre los niños y la tecnología y defender su tesis acerca de la educación mínimamente invasiva, el profesor de Tecnología Educativa de la Universidad de Newcastle, Sugata Mitra, creó el experimento "Hole in the Wall" (1999), en el cual se brindaba la oportunidad a niños de contextos desfavorecidos, que nunca habían tenido contacto con un ordenador, de relacionarse con este recurso de manera instintiva. Sugata Mitra y su equipo llegaron a la conclusión de que los niños solos son capaces de organizar y dirigir su propio aprendizaje en torno a un ordenador, además de aprender cómo compartir y enseñarse los unos a los otros.

En consecuencia con lo planteado hasta ahora, y tal y como ya defendía Negroponte (1995) con la generalización del uso de la tecnología se está produciendo una división de tipo generacional. Cada día, un mayor número de adultos está aprendiendo acerca de la tecnología a través de sus hijos, que les muestran formas de conocer e interactuar con el mundo real por medio de ella a partir del uso de Enciclopedias o diccionarios online, el CD-ROM o el libro electrónico. 
Y es que la informática ya no se ocupa de los ordenadores, sino de la vida misma. Hemos presenciado cómo los ordenadores se trasladan de enormes espacios con aire acondicionado a armarios, luego a escritorios y ahora a nuestras rodillas y a nuestros bolsillos. $Y$ este proceso aún no ha terminado (Negroponte, 1995, p.8).

Por tanto este estudio pretende reflexionar acerca de la evaluación de aquellos contenidos emocionales que se pueden derivar del uso de la tecnología, especialmente sobre aquellas aplicaciones educativas para dispositivos móviles destinadas al público infantil. En concreto, se analizan una serie de aplicaciones educativas que transmiten contenido emocional a partir de la clasificación de Gómez et al., (2000) sobre las dimensiones o competencias que sustentan la inteligencia emocional, basada a su vez en Goleman (1999). La primera dimensión es la competencia intrapersonal, que comprende el autoconocimiento, la destreza de identificar, reconocer y valorar las propias emociones; el autocontrol, la habilidad para controlar los propios impulsos y el desarrollo de una independencia conductual; la asertividad, que implica el saber expresar lo que se piensa, se siente, se desea o se necesita de manera clara y oportuna. Y por último, la proactividad, que desencadena la responsabilidad y disciplina propia de la persona, y se relaciona con la resolución de problemas y toma de decisiones. Otra gran competencia sería la interpersonal. Integrada por la empatía y las habilidades sociales. La empatía es la capacidad de tomar en cuenta diferentes maneras de pensar. Las habilidades sociales se clarifican en prácticas que implican la colaboración, el respeto hacia los demás, la tolerancia, la compasión y la confianza, y responsabilidad ante los otros. La última gran competencia implica un buen manejo del estrés y la capacidad de adaptación al cambio. Comprende las habilidades de automotivación y creatividad. La automotivación se identifica con el optimismo, tener metas según los propios intereses y poseer afán de superación. Por el contrario, la creatividad aporta flexibilidad, originalidad y voluntad de modificar o transformar la realidad.

En consonancia, se ha efectuado un estudio evaluativo apoyado en un instrumento (ficha de recogida de información) construido ad hoc para el análisis de app de contenido emocional organizada mediante la clasificación de las dimensiones emocionales mencionada anteriormente. Se presenta así los resultados obtenidos, así como la ficha con el fin de que puedan servir de base para posteriores investigaciones.

\section{MÉTODO}

El objetivo principal de este trabajo es realizar un análisis de aplicaciones educativas para dispositivos móviles con el fin de trabajar las emociones en niños en edad infantil, de 2 a 8 años. Partiendo de tal fin, se ha realizado un estudio descriptivo desde el punto de vista de la recogida de datos y análisis de los mismos, y un diseño de investigación de corte evaluativo. Para ello, se ha utilizado como instrumento una ficha de registro de información que ha permitido efectuar una recogida sistemática de información acerca del contenido emocional de dichas aplicaciones. En concreto, se han analizado las características, las propiedades, y perfiles de los potenciales destinatarios de estas aplicaciones, a la vez que se hace alusión a su contenido emocional y su influencia en el desarrollo integral de los niños.

Tras realizar un análisis de la bibliografía sobre el objeto de estudio, se realizó una primera fase consistente en elaboración y validación del instrumento de evaluación. Para llevar a cabo esta labor se ha diseñado una ficha de registro de información, en la que se exponen las dimensiones a analizar en cada una de las aplicaciones: Identificación, Contenido didácticoemocional y Diseño, con una escala tipo Likert de cinco categorías para favorecer la valoración de los ítems. Posteriormente dicho instrumento fue validado por medio de un juicio de expertos. La segunda fase, consistió en la selección de app de contenido emocional. Para su selección se establecieron una serie de criterios:

- Que ostentara alguna intención educativa, ya fuera para alcanzar el nivel de su etapa de progreso madurativo cognitivo $\mathrm{y} / \mathrm{o}$ emocional, $\mathrm{o}$ con el fin de que practicase ciertas competencias y/o habilidades que le ayudaran a su desarrollo integral.

- Estar disponibles en las principales plataformas de descarga de aplicaciones on-line del mercado actual. 
- Dirigirse al público infantil de entre 2 y 8 años.

- Garantizar la seguridad y privacidad de los menores, así como el no fomentar el acceso a contenido de adultos.

- Desarrollar algún tipo de contenido relacionado con el aprendizaje emocional: Autoconocimiento, autocontrol, automotivación, empatía, habilidades sociales, asertividad, proactividad y creatividad

Por último, en la tercera fase se procedió al análisis de las aplicaciones seleccionadas a través del instrumento construido y validado para este estudio.

\subsection{Muestra}

A la luz del proceso seguido se seleccionaron diez apps de contenido emocional para niños entre 2 y 8 años, que se presentan en la tabla 2.

Tabla 2. Muestra: Aplicaciones seleccionadas para su análisis

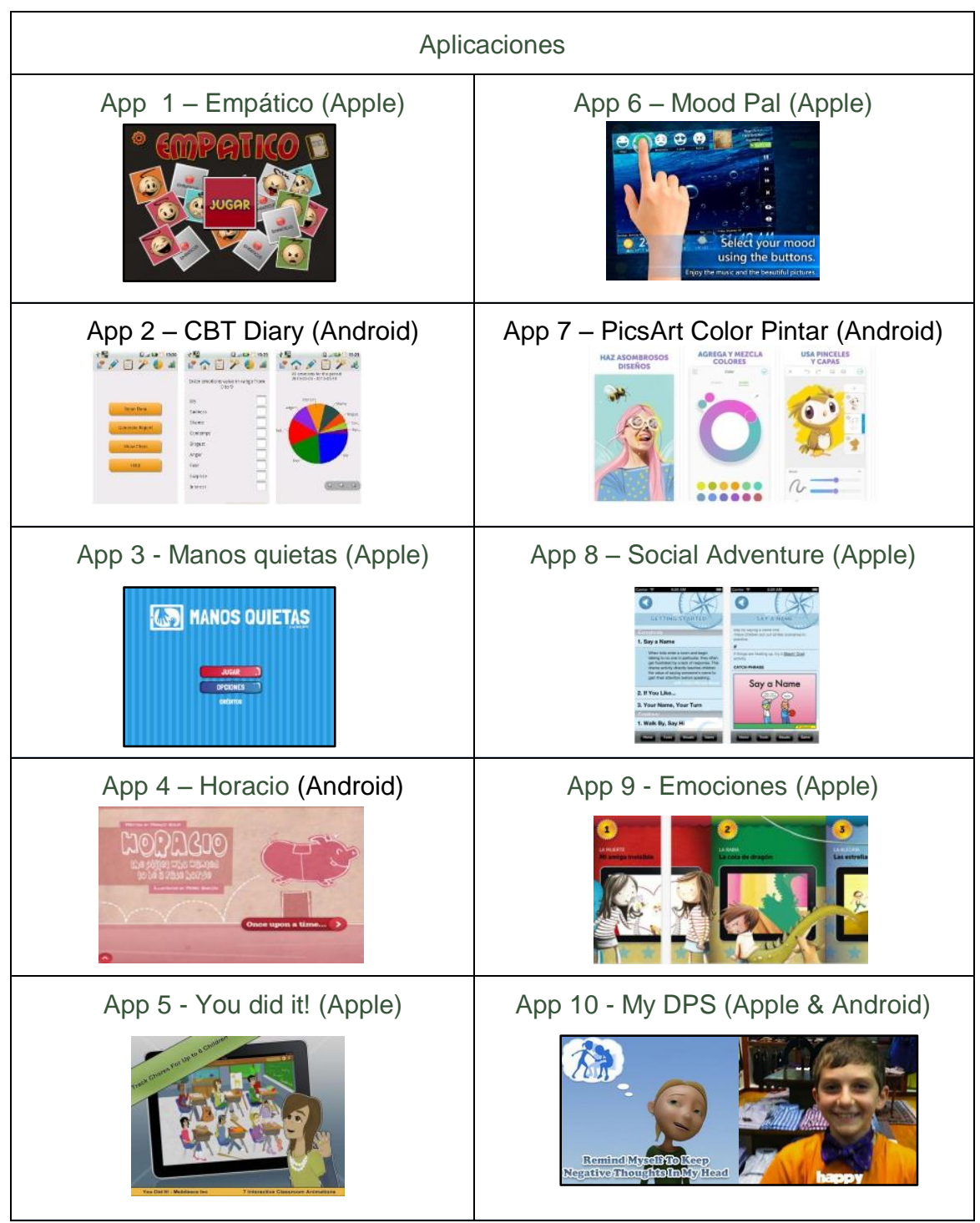




\subsection{Instrumento}

El instrumento realizado para analizar las aplicaciones seleccionadas consta de tres apartados según el tipo de aspectos sobre el que se pretende recabar información: identificación de la aplicación, su contenido emocional y su diseño. Estos primeros apartados permiten la clasificación de las dimensiones e ítems utilizados en cada uno de ellos.

En el primer apartado de la ficha (identificación) se recoge toda aquella información relevante acerca de las características propias de cada aplicación en cuanto a su identidad, tipo de soporte en el cual se reproduce, su localización, una breve descripción, los destinatarios para los cuales se ha realizado y la valoración media que los usuarios le otorgan.

El segundo gran apartado de esta ficha de observación es el que engloba las dimensiones que hacen alusión a las competencias generales de la inteligencia emocional (Goleman, 1999; Gómez et al., 2000) que se pueden obtener de la aplicación. Éstas se han dividido en tres grandes grupos para favorecer y facilitar su catalogación. Los ítems contenidos en cada uno de estos apartados se derivan de cada una de estas competencias. La competencia intrapersonal, integrada por el autoconocimiento, el autocontrol, la asertividad y la proactividad; la competencia interpersonal, que comprende la empatía y las habilidades sociales; y la última gran competencia, basada en un buen manejo del estrés y la capacidad de adaptación al cambio, englobando la automotivación y la creatividad.

El último apartado de la ficha es el correspondiente al diseño de la aplicación. Esta valoración se ha realizado utilizando como base la ficha de evaluación elaborada para tal fin basada en manuales de producción gráfica (Gutiérrez, 2005) y tratamiento de textos e imágenes (Johansson, Lundberg y Ryberg, 2004). En este apartado se ha de efectuar una valoración de la composición general del contenido. Se valora el tratamiento tipográfico, la legibilidad en cuanto al traking, kerning y largo de línea y el uso del color de las fuentes tipográficas utilizadas.

También por medio del tratamiento de la imagen se pretende realizar una valoración sobre la calidad técnica y estética de la aplicación aludiendo al uso que hace de frames, tablas o ventanas estructuradas, sistemas de navegación, sobre todo en cuanto a la posición de los botones, las barras de navegación o la existencia de menús de opciones y su funcionalidad. También se tienen en cuenta el uso de iconos y metáforas del entorno, al igual que se alude a la usabilidad de hipervínculos y pasos de pantalla. En este tratamiento de la imagen también se engloba al movimiento de ésta y la adecuada integración de medias, ralentización en la carga de la aplicación o se sus elementos, el tamaño de datos informáticos y su definición en las imágenes y el uso del color. La evaluación discrimina entre si se muestra una imagen real, real animada, ilustración o ilustración animada. En el caso de que el tipo de imagen contenida en la aplicación no posea estas u otras características se puede especificar en el apartado de observaciones. Otra de las particulares valorables es el contraste existente entre el texto y las imágenes con el fondo, con el fin de valorar la nitidez de ambos elementos. Además, crea distinción entre las funciones de anclaje, relevo u otro tipo entre el texto y la imagen.

En relación al análisis del contenido multimedia se ha de identificar el tipo de contenido que posee, ya sea sonido, vídeo, texto, gráficos u otros, y la relación que se puede apreciar entre cada uno de estos elementos. En cuanto a la usabilidad de la aplicación se analiza en función de la velocidad de las animaciones, lectura de datos, visualización y navegación. Al igual que se establece una valoración en cuanto al nivel de interacción con el usuario.

Por último, se ha de evaluar la concreción en las instrucciones del juego y/o actividad para determinar que éstas sean comprensibles por los usuarios. $Y$ en última estancia, se ha de establecer una valoración en cuanto a que la aplicación que está siendo objeto de análisis está adaptada a sus destinatarios. Para efectuar la valoración de cada uno de los ítems se utilizó una escala tipo Likert con cinco categorías: Muy mala (1); Mala (2); Regular (3); Buena (4); Muy buena (5). 
Este instrumento ha sido sometido a su validación por juicio de expertos. Con el fin de otorgarle un mayor grado de concreción y validez. En base a esta validación se tuvieron en cuenta las mejoras y propuestas en cuanto a un par de ítems del instrumento. Estas mejoras fueron básicamente el establecimiento de una mayor concreción a la hora de definir los ítems correspondientes acerca de la asertividad y el autocontrol en cuanto al contenido emocional que evalúa la ficha y la incorporación de un ítem que hiciera alusión a la adecuación del contenido y usabilidad a los usuarios.

La ficha de registro de información se encuentra publicada en pdf y doc, con licencia creative commons de reconocimiento, no comercial y compartir bajo la misma licencia, en la siguiente dirección: https://digitum.um.es/xmlui/handle/10201/54314 . Asimismo, se ha incluido como anexo a este artículo.

\section{RESULTADOS: VALORACIÓN Y CLASIFICACIÓN GENERAL DE LAS APPS DE CONTENIDO EMOCIONAL}

La realización de este estudio pretende poner de manifiesto importancia que poseen las emociones en el aprendizaje para lograr un desarrollo integral del niño. La información recabada a través la bibliografía existente incide en la necesidad de enfocar los procesos de aprendizaje de los niños en función del alcance de aquellas competencias que contiene la inteligencia emocional y no sólo las desarrolladas a través de procesos cognitivos, puesto que éstos son sólo una parte de su sistema de aprendizaje. Tras el análisis de las aplicaciones existentes para el desarrollo del contenido emocional se constata la amplia existencia de aplicaciones de corte educativo para poder llevar a cabo un proceso de aprendizaje emocional en niños. El análisis de la muestra de apps selecionadas permite concretar el processo que se puede seguir para realizar uma adecuada selección de apps que trabajen el contenido emocional en edad infantil.

Para ilustrar los resultados obtenidos del análisis de las aplicaciones se han creado dos tablas en las que se muestran de manera visual los resultados obtenidos en cuanto al análisis de las competencias emocionales y de diseño que ostenta cada aplicación. En la tabla 3 de Clasificación de apps, según la relación entre aplicación y emoción que trabaja, se han marcado aquellas emociones trabajadas en cada una de las apps. Mientras que en la tabla 4, en la que se incluye la valoración y clasificación general de los resultados obtenidos, se muestra una escala de ponderación ${ }^{*}$ (a pie de tabla) de las características técnicas y de diseño de dichas aplicaciones. Los resultados, recogidos en este artículo de forma sintética, están incluidos de forma ampliada en Franco (2017), donde se incluyen también los códigos y datos de identificación de todas las app analizadas.

En la tabla 3 se observa que las apps seleccionadas trabajan todas o algunas de las dimensiones de la inteligencia emocional. La app4 "Horacio", la app8 "Social Adventure" y la app9 "Emociones" permiten trabajar todas las dimensiones del contenido emocional mencionadas en este estudio, mientras que la app6 "Mood Pal" y la app7 "PicsArt Color Pintar" restringen su influencia como recurso para el aprendizaje emocional a tres competencias, en el caso de la app7, a la asertividad, la automotivación y la creatividad; y en el caso de la app6 a autoconocimiento 4, englobando también entre éstas el autoconocimiento. Exceptuando la app7 "PicsArt Color Pintar", antes mencionada, todas las aplicaciones trabajan alguna de las habilidades comprendidas dentro de las tres grandes dimensiones del aprendizaje emocional, la competencia intrapersonal, la interpersonal y la de manejo del estrés y la capacidad de adaptación. 
Tabla 3: Clasificación según la relación entre aplicación y emoción que trabaja

\begin{tabular}{|c|c|c|c|c|c|c|c|c|}
\hline \multicolumn{8}{|c|}{ CONTENIDO DIDÁCTICO-EMOCIONAL } & \\
\hline \multicolumn{4}{|c|}{ Intrapersonales } & \multicolumn{2}{|c|}{ Interpersonales } & \multicolumn{2}{|c|}{$\begin{array}{l}\text { Manejo del estrés y } \\
\text { capacidad de } \\
\text { adaptación }\end{array}$} & \\
\hline APP & Autoconocimiento & Autocontrol & Asertividad & Proactividad & Empatía & $\begin{array}{l}\text { Habilidades } \\
\text { sociales }\end{array}$ & Automotivación & Creatividad \\
\hline App1 & $x$ & & $\mathrm{X}$ & $x$ & $x$ & & $\mathrm{X}$ & \\
\hline App2 & $\mathrm{X}$ & $\mathrm{X}$ & $X$ & $\mathrm{X}$ & $\mathrm{X}$ & $\mathrm{X}$ & & \\
\hline App3 & & $\mathrm{X}$ & $\mathrm{X}$ & $\mathrm{X}$ & & & $\mathrm{x}$ & \\
\hline App4 & $\mathrm{x}$ & $X$ & $\mathrm{x}$ & $\mathrm{X}$ & $\mathrm{X}$ & $\mathrm{X}$ & $\mathrm{X}$ & $\mathrm{X}$ \\
\hline App5 & & $\mathrm{X}$ & & $\mathrm{X}$ & & & $x$ & $\mathrm{X}$ \\
\hline App6 & $x$ & & $\mathrm{X}$ & & & & $x$ & $\mathrm{X}$ \\
\hline App7 & & & $x$ & & & & $x$ & $X$ \\
\hline App8 & $x$ & $x$ & $x$ & $x$ & $x$ & $x$ & $x$ & $x$ \\
\hline App9 & $x$ & $X$ & $x$ & $X$ & $x$ & $X$ & $x$ & $x$ \\
\hline $\begin{array}{c}\text { App } 1 \\
0\end{array}$ & $X$ & $X$ & $X$ & $X$ & $x$ & & $x$ & $x$ \\
\hline
\end{tabular}

A continuación se presenta una tabla con los resultados de la valoración del diseño y características técnicas de las apps analizadas. Los resultados hallados ponen de manifiesto que la app7 "PicsArt Color Pintar" presenta muy buenas características estéticas y técnicas, basadas en la interacción, la creatividad, la accesibilidad y la usabilidad. Tanto la app2 "CBT Diary" como la app6 "Mood Pal" presentan un diseño y características técnicas poco atractivas y una estructura muy simple. La calidad técnica de la app6 es superior a la de la app2 en cuanto a que proporciona más probabilidades de interactuación con el programa. Sin embargo en la anterior se echa en falta esta posibilidad y un mayor grado de personalización por parte del usuario.

Tabla 4: Valoración del diseño y las características técnicas de las apps analizadas.

\begin{tabular}{|c|c|c|c|c|c|c|}
\cline { 2 - 7 } \multicolumn{1}{c|}{} & \multicolumn{7}{c|}{ DISEÑO Y CARACTERÍSTICAS TÉCNICAS } \\
\hline APP & $\begin{array}{c}\text { Composición } \\
\text { general }\end{array}$ & $\begin{array}{c}\text { Tratamiento } \\
\text { tipográfico }\end{array}$ & $\begin{array}{c}\text { Tratamiento de } \\
\text { la imagen }\end{array}$ & $\begin{array}{c}\text { Contenido } \\
\text { multimedia }\end{array}$ & $\begin{array}{c}\text { Concreción en las } \\
\text { instrucciones }\end{array}$ & $\begin{array}{c}\text { Adaptación a } \\
\text { destinatarios }\end{array}$ \\
\hline App1 & 5 & 4 & 5 & 4 & 5 & 5 \\
\hline App2 & 2 & 2 & 4 & 4 & 5 & 3 \\
\hline App3 & 5 & 5 & 4 & 5 & 5 & 5 \\
\hline App4 & 4 & 4 & 4 & 4 & 5 & 4 \\
\hline App5 & 4 & 4 & 5 & 4 & 4 & 3 \\
\hline App6 & 5 & 5 & 5 & 5 & 4 & 5 \\
\hline App7 & 5 & 5 & 5 & 5 & 5 & 5 \\
\hline App8 & 4 & 4 & 4 & 4 & 5 & 5 \\
\hline
\end{tabular}




\begin{tabular}{|c|l|l|l|l|l|l|}
\hline App9 & 5 & 5 & 5 & 4 & 5 & 5 \\
\hline App10 & 5 & 5 & 4 & 4 & 5 & 4 \\
\hline
\end{tabular}

("Valoración: 1: Muy Mala; 2: mala; 3: regular; 4: Buena; 5: Muy buena)

En general, las aplicaciones analizadas poseen una buena composición general que posibilita un aprendizaje fluido y didáctico. Las características más técnicas, como el tratamiento tipográfico, de la imagen y el contenido multimedia poseen una buena calidad pese a que algunas de las aplicaciones carecían de algún tipo de estas particularidades, como la falta de tipografía o de material interactivo. Sin embargo, todas las apps analizadas hacían un gran hincapié en especificar claramente sus instrucciones y, en su mayoría, adaptarse al nivel cognitivo, destreza y capacidad de sus destinatarios.

\section{DISCUSIÓN Y CONCLUSIONES}

Cada día existe más cantidad de material escrito sobre el desarrollo de la inteligencia a través de las TIC; sin embargo para contribuir al proceso educativo integral del niño también se ha de atender a la transmisión de contenidos de carácter emocional en su entorno más cotidiano. Y dado el gran auge y la expansión al alza de los dispositivos móviles y su uso educativo por parte de los menores resulta relevante el análisis de las aplicaciones de corte educacional sustentadas por dispositivos móviles que tienen como destinatarios el público infantil. Es por ello que sería un relevante campo de estudio para futuras investigaciones intentar establecer la conexión existente entre los diferentes ambientes educativos en los que los niños desarrollan un aprendizaje emocional, y los dispositivos en los que se transmite la información.

Como indican Nogueira y Ceinos (2015), el uso de la Tablet durante la etapa de crecimiento infantil puede facilitar el desarrollo cognitivo de la infancia, así como estimular la creatividad, el sentimiento de autonomía, la motivación, etc. Aun así, advierten que son las familias las que deben adquirir un compromiso real para regular el uso que sus hijos hacen de los dispositivos móviles, y por parte de la escuela, se debería promover iniciativas, a todas luces deseables de colaboración familia-escuela. Es tanto el interés que despierta este tema que los autores recogen las aportaciones de diferentes autores en relación con la aplicación de pautas, recomendaciones o decálogos para padres, madres y/o educadores sobre el uso de dispositivos tecnológicos por parte de los menores. Con el fin de que, como propone Morón (2010), se amplíe el marco de todos los aprendizajes, creando situaciones enriquecedoras y promoviendo que los niños sean los protagonistas directos de su propio aprendizaje.

En este sentido, más allá del análisis presentado en este artículo de apps de contenido emocional, consideramos que la ficha de registro de información elaborada ad hoc para esta investigación servirá para realizar evaluaciones con la intención de adaptar y mejorar el aprendizaje emocional del niño en su etapa escolar o en su contexto de ocio y entretenimiento, ya que se muestra como una herramienta para su uso tanto en el ámbito de aprendizaje formal como no formal. Conocemos el importante papel que juegan las emociones en el aprendizaje de los niños y hoy además sabemos que identificarlas posibilita a los educadores el poder orientarles en su desarrollo, que ellos mismos sepan identificarlas, fomentar la reflexión crítica y la toma de decisiones de forma autónoma, impulsar la creatividad por medio de elementos como la música o la imagen son algunas de las puestas en práctica que poseen estos recursos que cada día poseen más funcionalidad gracias al auge de los dispositivos móviles, herramientas con las que conviven los niños diariamente y a través de las cuales desarrollan también su aprendizaje. Las aplicaciones desarrolladas para estos dispositivos pretenden ser cada día más innovadoras y propiciar una mayor interacción entre el niño y su propio aprendizaje.

A partir del trabajo realizado en esta investigación de corte evaluativo se pretende crear un espacio web que sirva como sitio de referencia para catalogar estas y otras aplicaciones para el desarrollo de la inteligencia emocional en edad infantil disponibles en el mercado, utilizando como instrumento para su evaluación la ficha presentada en este artículo, recogida en el 
anexo. Asimismo, esta página web serviría para recoger experiencias de aprendizaje de la inteligencia emocional mediada por recursos tecnológicos. De esta manera sería posible orientar a padres, maestros, pedagogos y a toda la comunidad educativa en general sobre el contenido emocional que transmiten estas aplicaciones. La importancia de esta propuesta radica en que son dispositivos y recursos a partir de los cuales están aprendiendo los niños desde edades verdaderamente tempranas y es imperativo el análisis del contenido emocional que transmiten. Sin embargo, también se consideraría relevante llevar a cabo posteriores investigaciones, contando para ello, con un equipo multidisciplinar de expertos que puedan establecer, a partir del análisis cualitativo de aplicaciones y con la ficha de observación propuesta, coincidencias que aportaran una mayor fiabilidad a ésta y otras investigaciones acerca del contenido emocional y educativo de estas aplicaciones.

\section{REFERENCIAS BIBLIOGRÁFICAS}

AC Nielsen (2012). American Families See Tablets as Playmate, Teacher and Babysitter. Recuperado de: http://blog.nielsen.com/nielsenwire/online_mobile/american-familiesseetablets-as-playmate-teacher-and-babysitter/

Bar-On, R. (1997). The Emotional Quotient inventory (EQ-I): Techical Manual. Canadá: MultiHealth Systems.

Bisquerra, R. (2000). Educación emocional y bienestar. Barcelona: Praxis.

Cánovas, G. y otros. (2014). Menores de edad y conectividad en España: Tablets y Smartphones. PROTEGELES. Centro de Seguridad en Internet para los Menores en España.

Recuperado

de http://www.diainternetsegura.es/descargas/estudio movil smartphones tablets v2c.pdf

Comscore (2015) Global Digital Future in Focus. Recuperado de http://hispanictravelbloggers.com/wp-content/uploads/2016/08/Global-Future-in-FocusSPALA.pdf

Chia-Jung Lee (2011). Technology Integration and Emotional Learning. Recuperado de http://teachteachtech.coe.uga.edu/index.php/2011/05/13/technology-integration-andemotional-learning/

Crescenzi, L. (2014). Recepción, usos y hábitos mediáticos de los nativos digitales en edad preescolar, en Huertas, A., y Figueras, M. (eds). Audencias J uveniles; recepción, usos y hábitos Mediáticos. Bellaterra: Institut de la Comunicació. Universitat Autònoma de Barcelona. Recuperado de http://incom.uab.es/download/eBook InComUAB audienciajuvenilculturadigital.pdf

De La Barrera, M. L. y Donolo, D. (2009) Neurociencias y su importancia en contextos de aprendizaje. En Revista Digital Universitaria, 10 (4). Recuperado de http://www.revista.unam.mx/vol.10/num4/art20/int20.htm

Extremera N. y Fernández-Berrocal, P. (2003). La Inteligencia Emocional en el contexto educativo: hallazgos científicos de sus efectos en el aula (pp. 97-116). Revista de Educación, 332, 105.

Furger R. (2011). Digital Technology: Tools that Help Enhance Emotional Intelligence. En Edutopia.org. Recuperado de http://www.edutopia.org/digital-technology-tools-help-enhanceemotional-intelligence

Franco, S. (2017). Análisis de aplicaciones con contenido emocional para dispositivos móviles. Trabajo de Fin de Grado de Pedagogía de la Universidad de Murcia. Recuperado de https://digitum.um.es/xmlui/bitstream/10201/54423/1/Susana Franco Hernández App Emo cional.pdf 
Goleman, D. (1996). Inteligencia emocional. Barcelona: Kairós.

Goleman, D. (1999). La práctica de la inteligencia emocional. Barcelona: Kairós.

Gómez, J.M., Galiana, D. y León, D. (2000). "Que debes saber para mejorar tu empleabilidad". Elche: Universidad Miguel Hernández.

Gutiérrez García, B. (2005) Manual del proceso de Pre-Impresión. Aplicaciones en Quark-XPress, Photoshop y Freehand. Asturias: Nadetur.

Hole in the Wall. Mitra S. (1999). Recuperado de: http://www.hole-in-the-wall.com/index.html

Instituto Nacional de Estadística (INE) (2014). Encuesta sobre equipamiento y uso de tecnologías de información y comunicación en los hogares. Notas de prensa, 2 de octubre de 2014. Madrid: INE.

Johansson, K., Lundberg P., y Ryberg, R. (2004) Manual de Producción Gráfica. Recetas. Barcelona: Gustavo Gili

Kelly, R. (2010). A Learner-Centered, Emotionally Engaging Approach to Online Learning, Online Cl@ssroom, Junio (2009), 1. Recuperado de http://www.vcu.edu/cte/resources/newsletters archive/OC0906.pdf

León Barroso, H. (2012). Pantallas. De la Generación del Pulgar a la Generación del Indice, (pp. 177-181). En Teknocultura. Revista de Cultura Digital y Movimientos Sociales, 9(1), 180. Recuperado de http://dialnet.unirioja.es/descarga/articulo/3985571.pd

Matsumoto, M., Aliagas, C., Morgade, M., Correro, C., Galera, N., Roncero, C. y Póveda, D. (2016). Young Children (0-8) and Digital Technology. A qualitative exploratory study. Coordinated by the Joint Research Centre (JRC) European Commission. UAM y UAB. Recuperado de https://goo.gl/b7GGfg

Morón Macías, M. C. (2010). Una herramienta para aprender: El ordenador en las aulas de educación infantil, (pp. 1-11). En Temas para la Educación, revista digital para profesionales de la enseñanza, 9(58), $10 . \quad$ Recuperado de http://www2.fe.ccoo.es/andalucia/docu/p5sd7370.pdf

Negroponte, N. (1995). El Mundo Digital. Barcelona: Ediciones B.

Nogueira Pérez M. A. y Ceinos Sanz, C. (2015), Influencia de la tablet en el desarrollo infantil: Perspectivas y recomendaciones a tener en cuenta en la orientación familiar. Tendencias pedagógicas, 26, 42-46. Recuperado de: http://bit.ly/2057a6l

Prensky, M. (2006). Digital Natives, Digital Inmigrants: Do they really think differently? On the Horizon, (pp. 1-6) 9(6), $1 . \quad$ Recuperado de: http://www.marcprensky.com/writing/Prensky\%20-

\%20Digital\%20Natives,\%20Digital\%20Immigrants\%20-\%20Part2.pdf

Roig Vila, R. (2011). En Martínez Sánchez, F. y Solano Fernández, I. (Coord.) Comunicación y relaciones sociales de los jóvenes en la red. Murcia: Marfil. Universidad de Murcia.

Segers,E, Takke,L. y Verhoeven, L. (2004). Teacher-mediated versus computer-mediated storybook reading to children in native and multicultural kindergarten classrooms. School effectiveness and school improvement, 15 (2), 215-226.

Silverman, R. y Hines,S.(2009). The effects of multimedia-enhanced instruction on the vocabulary of english-language learners and non-english-language learners in prekindergarten through second grade. Journal of educational psychology, 101(2), 305-314. 
Van Scoter, J., Ellis, D., \& Railsback, J. (2001). What research says aboyt technology and child development. Technology in Early Childhood Education: Finding the Balance. (11- 15). Request Series: Portland.

Zabalza, M.A. (1987). Diseño y desarrollo curricular. Madrid: Narcea.

\section{INFORMACIÓN SOBRE LAS AUTORAS}

\section{Susana Franco Hernández Universidad de Murcia}

Graduada en Pedagogía por la Universidad de Murcia, especializada en edición, maquetación y diseño gráfico de materiales con fines educativos. Posee las titulaciones de Técnico Superior en Producción de Artes Gráficas y de Técnico en Preimpresión de productos gráficos y editoriales. Se ha dedicado profesionalmente a estos campos en diferentes empresas del sector, a la vez que participaba como voluntaria en programas para la formación y asesoramiento educativo a familias y talleres formativos organizados por la Concejalía de Bienestar Social y Sanidad del Ayuntamiento de Murcia.

\section{Isabel Ma Solano Fernández}

Universidad de Murcia

Doctora en Pedagogía por la Universidad de Murcia. Profesora de Tecnología Educativa del Departamento de Didáctica y Organización Escolar de la mencionada universidad. Miembro del Grupo de investigación Grupo de Investigación de Tecnología Educativa (GITE). Ha realizado estancias de investigación en la Universidad de Cambridge y en la London Metropolitan University (Reino Unido). Entre sus líneas de investigación destacan las siguientes: Recursos multimedia audiovisuales en contexto de enseñanza formal y no formal, La Educación Infantil en contextos enriquecidos con tecnologías, Entornos Personales de Aprendizaje, Metodología de la Investigación en Tecnología Educativa y estrategias metodológicas en contextos de enseñanza formal y no formal. Ha participado en proyectos de I+D nacionales e Internacionales relacionados con la implementación de las TIC en la sociedad y la escuela, lo que le ha permitido publicar en revistas y libros de impacto relacionados con la Tecnología Educativa.

\section{(cc) BY-NC}

Los textos publicados en esta revista están sujetos a una licencia de Reconocimiento 4.0 España de Creative Commons. Puede copiarlos, distribuirlos, comunicarlos públicamente y hacer obras derivadas siempre que reconozca los créditos de las obras (autoría, nombre de la revista, institución editora) de la manera especificada por los autores o por la revista. La licencia completa se puede consultar en:Licencia Creative Commons Atribución-NoComercial-Compartir por igual 4.0 Internacional. 